\title{
Eagle's Syndrome
}

\section{Thaís Gonçalves Pinheiro', Vítor Yamashiro Rocha Soares², Denise Bastos Lage Ferreira ${ }^{3}$, Igor Teixeira Raymundo 3 Luiz Augusto Nascimento ${ }^{4}$, Carlos Augusto Costa Pires de Oliveira ${ }^{5}$.}

1) MD. Fellowship in Laryngology, Department of Otorhinolaryngology, University of Sao Paulo.

2) MD. Ph.D. Student in Health Sciences, Department of Otorhinolaryngology - Head and Neck Surgery, University of Brasilia

3) MD. Attending Physician, Department of Otorhinolaryngology - Head and Neck Surgery, University of Brasilia

4) MD, PhD. Adjunct Professor, Department of Otorhinolaryngology - Head and Neck Surgery, University of Brasilia

5) MD, PhD. Full Professor, Department of Otorhinolaryngology - Head and Neck Surgery, University of Brasilia

Institution: University of Brasilia - University of Brasilia Hospital, Department of Otorhinolaryngology - Head and Neck Surgery SGAN, Via L2 Norte, Quadra 604/605, Asa Norte - Ambulatório II, Brasilia/DF - Brazil, ZC: 70.840-050

Mailing address: Vítor Yamashiro Rocha Soares - SGAN, Via L2 Norte, Quadra 604/605 - HUB - Anexo III, Apart. 20 - Asa Norte - Brasília/DF - Brazil, Zip code 70840-050 - E-mail: vyrsoares@gmail.com

Article received on October $5^{\text {th }}, 2011$. Article accepted on November $16^{\text {th }}, 2011$

\section{SUMMARY}

Introduction: Eagle's syndrome is characterized by cervicopharyngeal signs and symptoms associated with elongation of the styloid apophysis. This elongation may occur through ossification of the stylohyoid ligament, or through growth of the apophysis due to osteogenesis triggered by a factor such as trauma. Elongation of the styloid apophysis may give rise to intense facial pain, headache, dysphagia, otalgia, buzzing sensations, and trismus. Precise diagnosis of the syndrome is difficult, and it is generally confounded by other manifestations of cervicopharyngeal pain.

Objective: To describe a case of Eagle's syndrome.

Case Report: A 53-year-old man reported lateral pain in his neck that had been present for 30 years. Computed tomography (CT) of the neck showed elongation and ossification of the styloid processes of the temporal bone, which was compatible with Eagle's syndrome. Surgery was performed for bilateral resection of the stylohyoid ligament by using a transoral and endoscopic access route. The patient continued to present pain laterally in the neck, predominantly on his left side. CT was performed again, which showed elongation of the styloid processes. The patient then underwent lateral cervicotomy with resection of the stylohyoid process, which partially resolved his painful condition.

Final Comments: Patients with Eagle's syndrome generally have a history of chronic pain. Appropriate knowledge of this disease is necessary for adequate treatment to be provided. The importance of diagnosing this uncommon and often unsuspected disease should be emphasized, given that correct clinical-surgical treatment is frequently delayed. The diagnosis of Eagle's syndrome is clinical and radiographic, and the definitive treatment in cases of difficult-to-control pain is surgical.

Keywords: Neck Pain; Osteogenesis; Oral Surgical Procedures.

\section{INTRODUCTION}

Eagle's syndrome is characterized by cervicopharyngeal signs and symptoms associated with elongation of the styloid apophysis (1). The styloid apophysis consists of a bone projection originating in the tympanic portion of the temporal bone, which is approximately $25 \mathrm{~mm}$ in length (2). Elongation of the styloid apophysis may occur through ossification of the stylohyoid ligament, or through growth of the apophysis due to osteogenesis triggered by a factor such as trauma (1).

Elongation of the styloid apophysis or calcification of the stylohyoid ligament may give rise to intense facial pain, headache, dysphagia or odynophagia, otalgia, buzzing sensations, and trismus (1-4). Precise diagnosis of the syndrome is difficult. It is generally confounded by temporomandibular joint dysfunction, chronic tonsillitis, migraine, cluster headache, glossopharyngeal and trigeminal neuralgia, myofascial pain dysfunction syndrome, pain secondary to unerupted or impacted third molars, neck arthritis, and tumors of the base of the tongue. Finger palpation of the palatine tonsil increases the pain associated with Eagle's syndrome and corroborates its diagnosis. In addition to a clinical history and physical examination, radiological evaluation is also important for diagnosis (4).

The aims of this study were to describe a case of Eagle's syndrome followed up at the Otorhinolaryngology - Head and Neck Surgery Service and to conduct a critical review of the literature.

\section{CASE REPORT}

The patient was a 53-year-old man who reported having had dysphagia as well as a stabbing pain laterally in his neck and bilateral cervical pain in his 
temporomandibular joint for 30 years. The pain extended to the deltoid region and, although intermittent, occurred every day along with hardening of the left side of the neck and episodic frontal headache. The condition had progressively worsened over the preceding 10 years, in terms of both intensity and frequency. The patient reported that the pain worsened with heat, especially with exposure to sunlight, and when lying down on his back. Clinical treatment using analgesics, anti-inflammatory agents, or muscle relaxants did not alleviate the pain. The patient said that he had not had any previous pathological conditions, except for an appendectomy 32 years earlier. There were no palpable enlarged lymph nodes in the neck region. Oral examination showed a hardened lesion in the posterior tonsillar pillar measuring $15 \mathrm{~mm}$ and a retromolar bone spur on the left side.

Radiography and computed tomography of the neck showed elongation and ossification of the styloid processes of the temporal bone. The right styloid process measured $5.7 \mathrm{~cm}$ and the left measured $4.9 \mathrm{~cm}$, which was compatible with Eagle's syndrome (Figures 1A and 1B).

Surgery was performed for bilateral resection of the stylohyoid ligament by using a transoral endoscopic access route. However, the patient continued to present preauricular pain with a burning sensation irradiating to the sides of the neck and temporal region, predominantly on his left side. Another computed tomography scan was produced, which showed elongation and ossification of the styloid processes of the temporal bone, with the right side measuring $4.4 \mathrm{~cm}$ and the left side measuring $3.9 \mathrm{~cm}$. This suggested that the base of the styloid process continued to be present bilaterally. The patient then underwent lateral cervicotomy with resection of the stylohyoid process, which partially resolved his painful condition (Figures $1 \mathrm{C}$ and 1D).
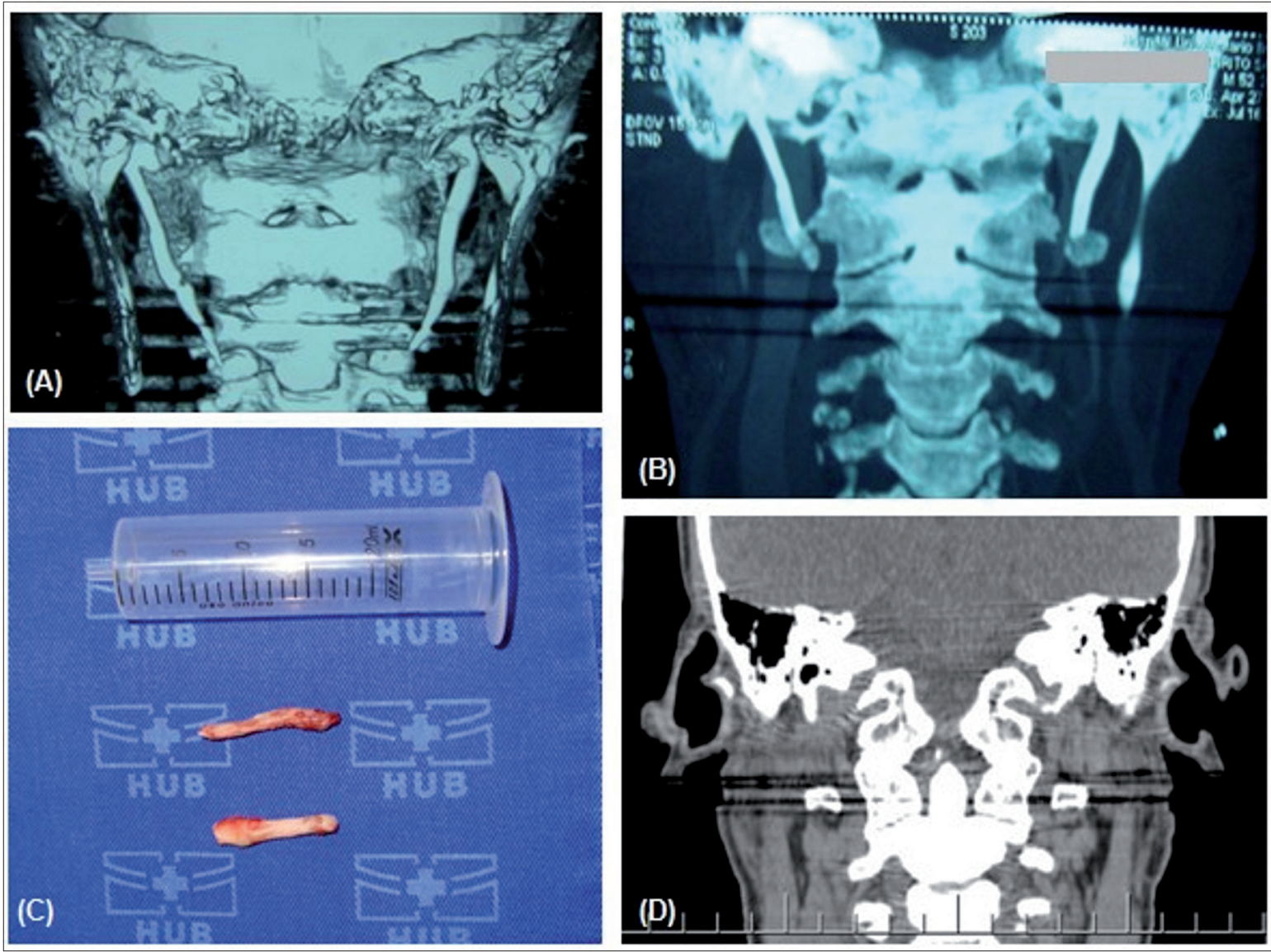

FIGURE 1. (A) Computed tomography (CT) scan of the neck showing elongation and ossification of the styloid processes of the temporal bone. The right styloid process measures $5.7 \mathrm{~cm}$ and the left measures $4.9 \mathrm{~cm}$, which is compatible with Eagle's syndrome. (B) CT scan of the neck after intraoral styloidectomy, showing ossification of the styloid processes. The right styloid process measures $4.4 \mathrm{~cm}$ and the left measures $3.9 \mathrm{~cm}$, thus suggesting that the base of the styloid process is present on both sides. (C) Surgical specimen after extraoral styloidectomy: right and left styloid processes. (D) CT scan of the neck after extraoral styloidectomy, showing the absence of elongation of the styloid processes on both sides. 


\section{DISCUSSION}

Eagle's syndrome is directly related to calcification of the stylohyoid ligament or elongation of the styloid process. These processes trigger symptoms such as recurrent facial pain, foreign body sensation, dysphagia, otalgia, and trismus (4). The styloid process, stylohyoid ligament, and lesser cornu of the hyoid bone form the stylohyoid complex, which originates embryologically in Reichert's cartilage of the second branchial arch $(1,2)$. Reichert's cartilage has 4 portions: the upper portion, from which the styloid process develops; the central portion, from which the stylohyoid ligament forms; the lower portion, from which the lesser cornu of the hyoid bone originates; and the basal portion, which gives rise to part of the hyoid bone $(1,4)$.

The reported sizes of the normal styloid process in the literature show a high degree of variability. Moffat et al conducted studies on cadavers and showed that the normal range of lengths of the styloid process was from $1.52 \mathrm{~cm}$ to $4.77 \mathrm{~cm}$ (5). In most radiographic studies, styloid processes are considered normal when they are shorter than $2.5 \mathrm{~cm}$ and elongated when they are longer than 4.0 $\mathrm{cm}$. In the latter situation, Eagle's syndrome is very likely to be present (1). It seems that there is little correlation between the extent of ossification and the severity of symptoms (6).

Several theories have been proposed to explain the elongation of the styloid process in Eagle's syndrome, including congenital elongation resulting from persistence of a mesenchymal embryonic leaf, with the capacity to produce bone tissue in adults; reactional ossifying hyperplasia due to osteitis, periostitis, or tendinitis, caused by surgical trauma or chronic local irritation; and ossification of the stylohyoid ligament related to endocrine disorders in women at menopause, accompanied by ossification of other ligaments. Some authors have affirmed that the syndrome frequently correlates with tonsillectomy (1). However, Prasad et al did not observe this relationship in a series of 58 cases (2). The patient in the present case did not have any previous pathological history of tonsillectomy.

The prevalence of elongation of the styloid process is variable. Some reports have shown that this abnormality occurs in $4 \%$ of the population, but that only $4 \%$ of such individuals present symptoms (2,4). In 1970, Kaufman et al confirmed this low rate of correlation, reporting that the styloid process was elongated in $7.3 \%$ of their patients (7). In 1979, Correl et al reported that calcification of the styloid complex or elongation of the styloid process was present in $18 \%$ of the 1700 panoramic radiographs that they examined, and observed low concordance with the presence of symptoms (8). Thus, the majority of patients with an elongated styloid process are asymptomatic (4). Greater prevalence of the disease has been found in women (3:1) $(2,4)$.

The main symptoms of the syndrome include nonspecific neck pain, foreign body sensation in the pharynx, odynophagia, otalgia, pain in the temporomandibular joint, and trismus (2). Painful palpation of the tonsillar fossa, together with suggestive clinical and radiological signs, corroborates the diagnosis of Eagle's syndrome. In addition, pain relief achieved through infiltration of local anesthetics into the tonsillar fossa is suggestive of the disease $(3,6)$.

Radiological examination can confirm the syndrome. Radiography can show the increased length of the styloid process. Currently, computed tomography is considered to be the best examination for defining the length and angulation of the styloid process, and also enables an evaluation of the anatomical relationships between the stylohyoid complex and adjacent structures $(1,3,4)$. According to some authors, panoramic or lateral radiographs are sufficient for diagnosing the disease (2).

The other conditions identified in differential diagnosis for the syndrome include temporomandibular joint dysfunction, pain secondary to unerupted or impacted third molars, glossopharyngeal and trigeminal neuralgia, migraine, neck arthritis, cluster headache, myofascial pain dysfunction syndrome, and tumors (4). Neuralgia of the $\mathrm{IX}^{\text {th }}$ and $\mathrm{V}^{\text {th }}$ cranial pairs consists of sudden sharp pain of short duration that arises through stimulation of the trigger zone, which is unlike that observed in Eagle's syndrome, where the pain is dull and persistent. Disorders of the temporomandibular joint and third molar pain are confirmed through radiological examinations (1).

Treatment for Eagle's syndrome can be surgical, but is not always. Pain control may be achieved in some patients by means of oral analgesics. Some authors have suggested that transpharyngeal infiltration of steroids and local anesthetics in the tonsillar fossa should be used $(1,2)$. The treatment of choice is styloidectomy, which can be performed transorally or extraorally. The transoral route consists of an incision in the tonsillar fossa, after tonsillectomy if necessary, which is followed by identification, separation, and excision of the styloid process. In the external technique, an incision is made in the neck, in the region proximal to the sternocleidomastoid muscle, into the hyoid bone, followed by identification, separation, and removal of the styloid process. This technique makes it possible to view the structures better, thus enabling greater resection of the styloid process (1) with less chance of deep infection in the neck (2). However, the extraoral technique requires a longer surgical recovery and it involves greater morbidity 
(relating to drains, sutures, risk of facial nerve lesions, and scarring of the neck).

\section{FINAL COMMENTS}

Patients with Eagle's syndrome generally have a history of chronic pain treated by several physicians. Appropriate knowledge of this disease is necessary for adequate treatment to be provided. The importance of diagnosing this uncommon and often unsuspected disease should be emphasized, given that correct clinical-surgical treatment is frequently delayed. The diagnosis of Eagle's syndrome is clinical and radiographic, and the definitive treatment in cases of difficult-to-control pain is surgical.

\section{REFERENCES}

1. Fini G, Gasparini G, Filippini F, Becelli R, Marcotullio D. The long styloid process syndrome or Eagle's syndrome. J Craniomaxillofac Surg. 2000;28:123-7.

2. Prasad KC, Kamath MP, Reddy JM, Raju K, Agarwal S. Elongated styloid process (Eagle's syndrome): a clinical study. J Oral Maxillofac Surg. 2002;60(2):171-5.
3. Beder E, Ozgursoy OB, Ozgursoy SK, Anadolu Y. Threedimensional computed tomography and surgical treatment for Eagle's syndrome. Ear Nose Throat J. 2006;85(7):4435 .

4. Mendelsohn AH, Berke GS, Chhetri DK. Heterogeneity in the clinical presentation of Eagle's syndrome. Otolaryngol Head Neck Surg. 2006;134:389-93.

5. Moffat DA, Ramsden RT, Shaw HJ. The styloid process syndrome: aetiological factors and surgical management. J Laryngol Otol. 1977;91(4):279-94.

6. Diamond LH, Cottrell DA, Hunter MJ, Papageorge M. Eagle's syndrome: a report of 4 patients treated using a modified extraoral approach. J Oral Maxillofac Surg. 2001;59:1420-6.

7. Kaufman SM, Elzay RP, Irish EF. Styloid process variation. Radiologic and clinical study. Arch Otolaryngol. 1970;91(5):460-3.

8. Correl R, Jensen J, Taylor J, et al. Mineralization of the stylohyoid-stylomandibular ligament complex: A radiographic incidence study. Oral Surg Oral Med Oral Pathol. 1979; 48:286-91. 